\title{
Propagation in Diagonal Anisotropic Chirowaveguides
}

\author{
S. Aib, ${ }^{1}$ F. Benabdelaziz, ${ }^{1}$ C. Zebiri, ${ }^{2}$ and D. Sayad ${ }^{3}$ \\ ${ }^{1}$ Department of Electronics, University Mentouri of Constantine, Constantine, Algeria \\ ${ }^{2}$ Department of Electronics, University Ferhat Abbas of Sétif, Sétif, Algeria \\ ${ }^{3}$ Department of Electrical Engineering, University of 20 Aout 1955, Skikda, Algeria
}

Correspondence should be addressed to S. Aib; sammalak3@yahoo.com

Received 29 August 2016; Revised 30 December 2016; Accepted 22 February 2017; Published 8 March 2017

Academic Editor: Armin Gerhard Aberle

Copyright (C) 2017 S. Aib et al. This is an open access article distributed under the Creative Commons Attribution License, which permits unrestricted use, distribution, and reproduction in any medium, provided the original work is properly cited.

\begin{abstract}
A theoretical study of electromagnetic wave propagation in parallel plate chirowaveguide is presented. The waveguide is filled with a chiral material having diagonal anisotropic constitutive parameters. The propagation characterization in this medium is based on algebraic formulation of Maxwell's equations combined with the constitutive relations. Three propagation regions are identified: the fast-fast-wave region, the fast-slow-wave region, and the slow-slow-wave region. This paper focuses completely on the propagation in the first region, where the dispersion modal equations are obtained and solved. The cut-off frequencies calculation leads to three cases of the plane wave propagation in anisotropic chiral medium. The particularity of these results is the possibility of controlling the appropriate cut-off frequencies by choosing the adequate physical parameters values. The specificity of this study lies in the bifurcation modes confirmation and the possible contribution to the design of optical devices such as high-pass filters, as well as positive and negative propagation constants. This negative constant is an important feature of metamaterials which shows the phenomena of backward waves. Original results of the biaxial anisotropic chiral metamaterial are obtained and discussed.
\end{abstract}

\section{Introduction}

Bianisotropic materials are special types of media where the physical parameters properties (permittivity, permeability, and magnetoelectric parameters) are tensors. They are characterized by constitutive equations that present a coupling between electric and magnetic fields [1]. These materials exhibit interesting applications in electromagnetic wave propagation [2-4].

Indeed, the chiral is a subset of bianisotropic medium case. By definition, chirality is purely a geometrical notion, which is due to the lack of bilateral symmetry of an object [2]. So, the chiral object is a three-dimensional body that cannot be superposed on its mirror image by translation or rotation [2]. Furthermore, chirality concept leads to left and right waves notions where RCP (i.e., right circularly polarized) and LCP (i.e., left circularly polarized) each has a different refractive index and phase velocity. The two corresponding refractive indices are $n_{ \pm}=\sqrt{\mu_{r} \varepsilon_{r}} \pm \xi$ [5] $\left(\mu_{r}\right.$ : relative permeability, $\varepsilon_{r}$ : relative permittivity).

In fact, anisotropic chiral medium plays a crucial role by having negative refractive index (left-handed) materials that has opened new horizons in optics and becomes subject of important scientific interests [6-9]. It was stated through theoretical and experimental results that anisotropic chiral media can have a negative refraction index like isotropic media with both negative permittivity and permeability. The negative refractive index can be reached by either increasing the chirality parameter or operating near the electric and/or magnetic resonance frequency zones, where the value of $\sqrt{\mu_{r} \varepsilon_{r}}$ becomes smaller than the chirality parameter value $\xi$, which becomes strong around the resonance frequencies, as reported in $[10,11]$. Generally, natural chiral materials, such as quartz and sugar solution, have $\xi<1$ and $\sqrt{\mu_{r} \varepsilon_{r}}>1$, so negative refraction is not possible in these materials [12]. However, with artificial chiral metamaterials, macroscopic parameters can be clearly identified. Moreover, the notion of chiral nihility, when the values of $\mu_{r}$ and $\varepsilon_{r}$ of the medium are small and very close to zero, makes the refraction index negative for one of the circular polarization modes, even when $\xi$ is small $[5,13]$. In addition, it is reported that it is simpler to achieve and realize negative refraction in chiral materials than with regular metamaterials [14]. 


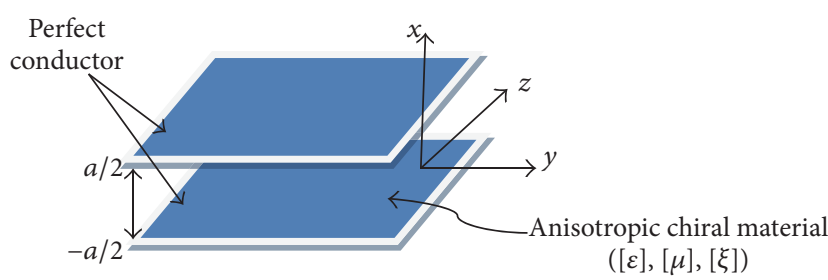

FIgURE 1: Parallel plate waveguide filled with anisotropic chiral material.

In this work, a special interest is given to the wave propagation study in chiral-core waveguide due to its different physical parameters diversity. In this scope, wave propagation in anisotropic chiral medium is modeled and studied where tensors of chirality, permittivity, and permeability are diagonal. The A-formalism [15] related to the proposed structure is used to facilitate the analytical calculation procedure of Maxwell's equations. Curves of normalized propagation constants are plotted with respect to normalized frequency, where positive and negative propagation constants are presented.

\section{Formulation of the Problem}

In this section, we analyze the parallel plate chirowaveguide depicted in Figure 1 with infinite perfectly conducting planes placed at $x= \pm a / 2$. The chirowaveguide propagation direction is along $z$-axis, whereas the field quantities are all independent of $y$-axis [2].

In general, bianisotropic medium is characterized by the following constitutive equations, as presented in [15]:

$$
\begin{aligned}
B & =[\mu] H+([\chi]+j[\xi]) \sqrt{\mu_{0} \varepsilon_{0}} E, \\
D & =[\varepsilon] E+([\chi]-j[\xi]) \sqrt{\mu_{0} \varepsilon_{0}} H,
\end{aligned}
$$

where $E, H, D$, and $B$ are, respectively, the electric field, the magnetic field, the electric flux density, and the magnetic flux density. $[\varepsilon]$ and $[\mu]$ are, respectively, the electric permittivity and the magnetic permeability tensors. $\varepsilon_{0}$ and $\mu_{0}$ are the free space permittivity and permeability, respectively. $[\chi]$ is the nonreciprocity (Tellegen) tensor and $[\xi]$ is the chirality (Pasteur) tensor. In fact, this study is based on Pasteur medium, which is a reciprocal anisotropic chiral, (i.e., $[\chi]=0$ and $[\xi] \neq 0)$. Hence, (1) becomes

$$
\begin{aligned}
& B=[\mu] H+j[\xi] \sqrt{\mu_{0} \varepsilon_{0}} E, \\
& D=[\varepsilon] E-j[\xi] \sqrt{\mu_{0} \varepsilon_{0}} H,
\end{aligned}
$$

and the permittivity, permeability, and chirality tensors of the considered medium are

$$
[\varepsilon]=\left[\begin{array}{ccc}
\varepsilon_{x} & 0 & 0 \\
0 & \varepsilon_{y} & 0 \\
0 & 0 & \varepsilon_{z}
\end{array}\right],
$$

$$
\begin{aligned}
& {[\mu]=\left[\begin{array}{ccc}
\mu_{x} & 0 & 0 \\
0 & \mu_{y} & 0 \\
0 & 0 & \mu_{z}
\end{array}\right],} \\
& {[\xi]=\left[\begin{array}{ccc}
\xi_{x} & 0 & 0 \\
0 & \xi_{y} & 0 \\
0 & 0 & \xi_{z}
\end{array}\right] .}
\end{aligned}
$$

After substantial algebraic manipulations of Maxwell's equations, considering the constitutive equation, we obtain the following set of coupled differential equations of the $z$ components of the electric and magnetic fields:

$$
\begin{gathered}
\frac{\partial^{2}}{\partial x^{2}}\left\{\begin{array}{l}
E_{z} \\
H_{z}
\end{array}+\left(\left(\omega^{2} \mu_{z} \varepsilon_{z}+\beta_{0}^{2} \xi_{z}^{2}\right)-\beta^{2}\right)\left\{\begin{array}{l}
E_{z} \\
H_{z}
\end{array}\right.\right. \\
\mp j 2 \omega^{2} \sqrt{\mu_{0} \varepsilon_{0}} \xi_{z}\left\{\begin{array}{l}
\mu_{z} H_{z} \\
\varepsilon_{z} E_{z}
\end{array}=0 .\right.
\end{gathered}
$$

The examination of these two coupled equations shows that $\xi_{z}$ is the only coupling parameter which enables the appearance of the bifurcated modes. The cancellation of this parameter suppresses coupling even with the presence of the other parameters of chirality $\xi_{x}$ and $\xi_{y}$

Let

$$
\begin{aligned}
H_{z}^{\prime} & =\sqrt{\frac{\mu_{z}}{\varepsilon_{z}}} H_{z}, \\
\left(E_{z}+j H_{z}^{\prime}\right) & =A_{z}, \\
\left(E_{z}-j H_{z}^{\prime}\right) & =B_{z} .
\end{aligned}
$$

The following decoupled equations are obtained:

$$
\begin{aligned}
& \frac{\partial^{2} A_{z}}{\partial x^{2}}+\left(\kappa_{+z}^{2}-\beta^{2}\right) A_{z}=0, \\
& \frac{\partial^{2} B_{z}}{\partial x^{2}}+\left(\kappa_{-z}^{2}-\beta^{2}\right) B_{z}=0,
\end{aligned}
$$

where

$$
\begin{aligned}
& \kappa_{+i}=\left(\omega \sqrt{\mu_{i} \varepsilon_{i}}-\beta_{0} \xi_{i}\right)=\beta_{0}\left(\sqrt{\mu_{i} \varepsilon_{i}}-\xi_{i}\right)=\beta_{0} n_{+i}, \\
& \kappa_{-i}=\left(\omega \sqrt{\mu_{i} \varepsilon_{i}}+\beta_{0} \xi_{i}\right)=\beta_{0}\left(\sqrt{\mu_{i} \varepsilon_{i}}+\xi_{i}\right)=\beta_{0} n_{-i}
\end{aligned}
$$

with $\xi_{i}<0, i=x, y$, and $z$.

$\kappa_{ \pm i}$ are the right and left wave numbers. $n_{ \pm i}$ are the refractive index of RCP and LCP plane wave. $\beta_{0}$ and $\beta$ are the free space and medium propagation constants, respectively.

Let us take

$$
\begin{aligned}
& U_{1}=\sqrt{\left(\kappa_{+z}^{2}-\beta^{2}\right)}, \\
& U_{2}=\sqrt{\left(\kappa_{-z}^{2}-\beta^{2}\right)} .
\end{aligned}
$$


TABLE 1: Conditions and solutions for the chiral three regions.

\begin{tabular}{lccc}
\hline & Cases & $\begin{array}{c}\text { Conditions and } \\
\text { velocities }\end{array}$ & Types of solutions \\
Fast-fast-wave region & $U_{1,2}=\sqrt{\left(\kappa_{ \pm z}^{2}-\beta^{2}\right)}$ & $\beta<\kappa_{-z}<\kappa_{+z}$ & $A_{z}=G_{1} \cos \left(U_{1} x\right)+G_{2} \sin \left(U_{1} x\right)$ \\
& $v_{p}>v_{\mathrm{RCP}}>v_{\mathrm{LCP}}$ & $B_{z}=M_{1} \cos \left(U_{2} x\right)+M_{2} \sin \left(U_{2} x\right)$ \\
Fast-slow-wave region & $U_{1}=\sqrt{\left(\kappa_{+z}^{2}-\beta^{2}\right)}$ & $\kappa_{-z}<\beta<\kappa_{+z}$ & $A_{z}=G_{1} \cos \left(U_{1} x\right)+G_{2} \sin \left(U_{1} x\right)$ \\
& $U_{2}=\sqrt{\left(\beta^{2}-\kappa_{-z}^{2}\right)}$ & $v_{\mathrm{LCP}}>v_{p}>v_{\mathrm{RCP}}$ & $B_{z}=M_{1} \cosh \left(U_{2} x\right)+M_{2} \sinh \left(U_{2} x\right)$ \\
Slow-slow-wave region & $U_{1,2}=\sqrt{\left(\beta^{2}-\kappa_{ \pm z}^{2}\right)}$ & $\begin{array}{c}\kappa_{-z}<\kappa_{+z}<\beta \\
v_{\mathrm{LCP}}>v_{\mathrm{RCP}}>v_{p}\end{array}$ & $A_{z}=G_{1} \cosh \left(U_{1} x\right)+G_{2} \sinh \left(U_{1} x\right)$ \\
\hline
\end{tabular}

$v_{p}$ is the waveguide phase velocity; $v_{\mathrm{LCP}}$ and $v_{\mathrm{RCP}}$ are the LCP and RCP velocities, respectively, along $z$-axis. $G_{1}, G_{2}, M_{1}$, and $M_{2}$ are constants.

The solutions of the differential equations (6) are given in Table 1, taking into account the three cases: fast-fast-wave, fast-slow-wave, and slow-slow-wave regions imposed by the chiral medium [2].

In this work, we deal only with the first case (fast-fastwave region):

$$
\begin{gathered}
A_{z}=G_{1} \cos \left(U_{1} x\right)+G_{2} \sin \left(U_{1} x\right), \\
B_{z}=M_{1} \cos \left(U_{2} x\right)+M_{2} \sin \left(U_{2} x\right) .
\end{gathered}
$$

The longitudinal $E_{z}$ and transversal $E_{y}$ components can be expressed as follows:

$$
\begin{aligned}
E_{z}= & \frac{1}{2}\left(A_{z}+B_{z}\right) \\
= & \frac{G_{1}}{2} \cos \left(U_{1} x\right)+\frac{G_{2}}{2} \sin \left(U_{1} x\right)+\frac{M_{1}}{2} \cos \left(U_{2} x\right) \\
& +\frac{M_{2}}{2} \sin \left(U_{2} x\right) \\
E_{y}= & -\frac{1}{2} c_{1} \cdot G_{1} \sin \left(U_{1} x\right)+\frac{1}{2} c_{1} \cdot G_{2} \cos \left(U_{1} x\right)+\frac{1}{2} c_{2} \\
& \cdot M_{1} \sin \left(U_{2} x\right)-\frac{1}{2} c_{2} \cdot M_{2} \cos \left(U_{2} x\right)
\end{aligned}
$$

with

$$
\begin{aligned}
& c_{1}=\frac{\kappa_{+x} U_{1}}{\left(\kappa_{+x} \kappa_{+y}-\beta^{2}\right)}, \\
& c_{2}=\frac{\kappa_{-x} U_{2}}{\left(\kappa_{-x} \kappa_{-y}-\beta^{2}\right)} .
\end{aligned}
$$

The propagation constant is supposed to be a real-valued quantity. The boundary conditions imposed by the adopted structure $[2,16,17]$ are

$$
\begin{aligned}
E_{z} & =0 \longrightarrow \\
x & = \pm \frac{a}{2}, \\
E_{y} & =0 \longrightarrow \\
x & = \pm \frac{a}{2},
\end{aligned}
$$

where $a$ is the chiral material thickness.

The enforcement of these conditions leads to the following $4 \times 4$ matrix system equation:

$$
\left[\begin{array}{cccc}
\cos \left(\frac{U_{1} a}{2}\right) & \sin \left(\frac{U_{1} a}{2}\right) & \cos \left(\frac{U_{2} a}{2}\right) & \sin \left(\frac{U_{2} a}{2}\right) \\
\cos \left(\frac{U_{1} a}{2}\right) & -\sin \left(\frac{U_{1} a}{2}\right) & \cos \left(\frac{U_{2} a}{2}\right) & -\sin \left(\frac{U_{2} a}{2}\right) \\
-c_{1} \sin \left(\frac{U_{1} a}{2}\right) & c_{1} \cos \left(\frac{U_{1} a}{2}\right) & +c_{2} \sin \left(\frac{U_{2} a}{2}\right) & -c_{2} \cos \left(\frac{U_{2} a}{2}\right) \\
c_{1} \sin \left(\frac{U_{1} a}{2}\right) & c_{1} \cos \left(\frac{U_{1} a}{2}\right) & -c_{2} \sin \left(\frac{U_{2} a}{2}\right) & -c_{2} \cos \left(\frac{U_{2} a}{2}\right)
\end{array}\right]\left[\begin{array}{l}
G_{1} \\
G_{2} \\
M_{1} \\
M_{2}
\end{array}\right]=0
$$


This system has a nontrivial solution only if the following equations are satisfied:

$$
\begin{aligned}
\Delta_{1,2}= & \left(\frac{1}{c_{1}}+\frac{1}{c_{2}}\right) \sin \left(\frac{U_{1} a}{2}+\frac{U_{2} a}{2}\right) \\
& \pm\left(\frac{1}{c_{1}}-\frac{1}{c_{2}}\right) \sin \left(\frac{U_{1} a}{2}-\frac{U_{2} a}{2}\right)=0 .
\end{aligned}
$$

The solutions of the above equations lead to two modes: RCP and LCP.

\section{Results and Discussions}

In order to investigate the propagation characteristics in the anisotropic chiral medium, different types of chiral medium are chosen considering various values of the physical parameters.

3.1. Bi-Isotropic Case. In bi-isotropic case, the chirality parameter $\xi$, permeability $\mu$, and permittivity $\varepsilon$ are scalars; the dispersion equation (14) becomes

$$
\begin{gathered}
\Delta_{1,2}=\left(\frac{\sqrt{\kappa_{+}^{2}-\beta^{2}}}{\kappa_{+}}+\frac{\sqrt{\kappa_{-}^{2}-\beta^{2}}}{\kappa_{-}}\right) \\
\cdot \sin \left(\frac{\sqrt{\kappa_{+}^{2}-\beta^{2}} a}{2}+\frac{\sqrt{\kappa_{-}^{2}-\beta^{2}} a}{2}\right) \\
\pm\left(\frac{\sqrt{\kappa_{+}^{2}-\beta^{2}}}{\kappa_{+}}-\frac{\sqrt{\kappa_{-}^{2}-\beta^{2}}}{\kappa_{-}}\right) \\
\cdot \sin \left(\frac{\sqrt{\kappa_{+}^{2}-\beta^{2}} a}{2}-\frac{\sqrt{\kappa_{-}^{2}-\beta^{2}} a}{2}\right)=0 .
\end{gathered}
$$

The cut-off frequencies are

$$
f_{c}=\frac{n}{2 a \cdot \sqrt{\varepsilon \mu}}
$$

and we obtain exactly the same dispersion equation and cutoff frequencies as reported in [2], where $\Omega=\omega \cdot a \sqrt{\varepsilon_{0} \mu_{0}} \Rightarrow$ $\Omega / 2 \pi=f \cdot a \sqrt{\varepsilon_{0} \mu_{0}}$ is the normalized frequency.

Our results for the simple case (chiral isotropic medium) shown in Figure 2 are in good agreement with those presented in [2]; this confirms our calculations. The bifurcated modes (LCP and RCP) are well distinguished and start from the same cut-off frequencies; this is an essential feature of chiral material.

As illustrated in Figure 3, the effect of the chirality on the RCP and LCP modes of the first mode is quite different. For the first one (Figure 3(a)), the RCP mode decreases keeping the same shape until the condition $\sqrt{\mu_{r} \varepsilon_{r}}>|\xi|$ is no more satisfied for $\xi \geq 3 * \xi_{p}$ where the mode becomes evanescent $\left(\alpha_{\mathrm{RCP}}=j \beta_{\mathrm{RCP}}\right)$, whereas, in the second mode (Figure $\left.3(\mathrm{~b})\right)$, the LCP one becomes quasi-constant with the increase of $\xi$

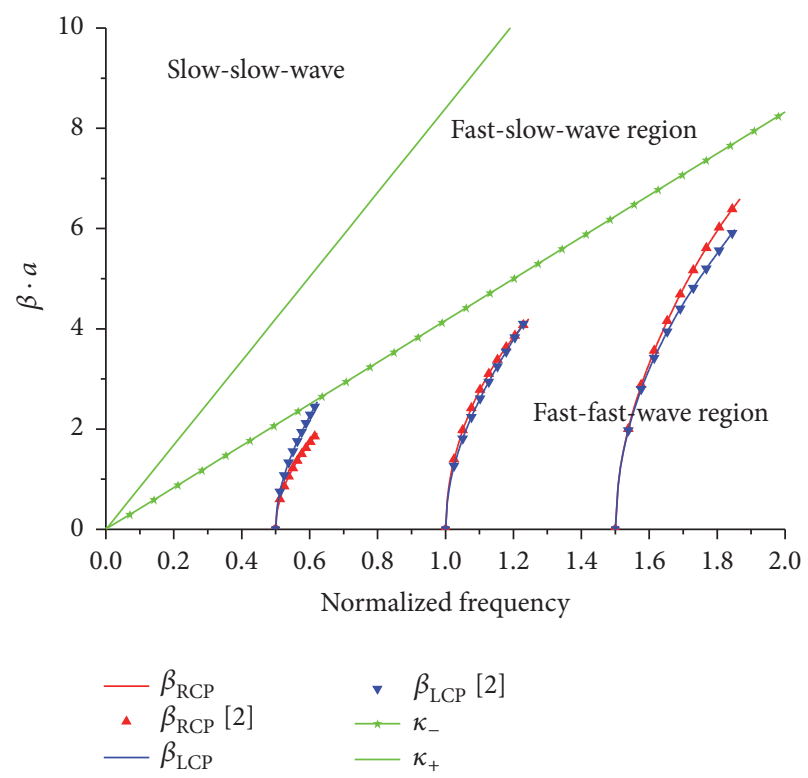

FIGURE 2: RCP and LCP propagation constants in a parallel plate chirowaveguide in fast-fast-wave region with $\varepsilon=\varepsilon_{0}, \mu=\mu_{0}$, and $\xi=-0.337$.

TABLE 2: Three cases of cut-off frequencies values according to conditions on physical parameters.

\begin{tabular}{lcc}
\hline Cases & $\begin{array}{c}\text { Conditions on physical } \\
\text { parameters }\end{array}$ & Cut-off frequency value \\
\hline $\begin{array}{l}\text { First } \\
\text { case }\end{array}$ & $\xi_{z} \sqrt{\mu_{r y} \varepsilon_{r y}}=\xi_{y} \sqrt{\mu_{r z} \varepsilon_{r z}}$ & $f_{c}=\frac{n}{2 \sqrt{\mu_{z} \varepsilon_{z} a}}$ \\
Second & $\sqrt{\mu_{r z} \varepsilon_{r z}} \neq\left|\xi_{z}\right|$ & \\
case & $\sqrt{\mu_{r y} \varepsilon_{r y}} \sqrt{\mu_{r z} \varepsilon_{z}}=\xi_{z} \xi_{y}$ & $f_{c}=\frac{n}{2 \sqrt{\varepsilon_{0} \mu_{0}} \xi_{z} a}$ \\
& $\sqrt{\mu_{r z} \varepsilon_{r z}} \neq\left|\xi_{z}\right|$ & $f_{c}=\frac{n}{2 \sqrt{\mu_{z} \varepsilon_{z} a}}=$ \\
Third & $\xi_{z} \sqrt{\mu_{r y} \varepsilon_{r y}} \neq \xi_{y} \sqrt{\mu_{r z} \varepsilon_{z}}$ & $\frac{n^{\prime}}{2 \sqrt{\varepsilon_{0} \mu_{0}} \xi_{z} a}$ with \\
case & $\sqrt{\mu_{r y} \varepsilon_{r y}} \sqrt{\mu_{r z} \varepsilon_{z}} \neq \xi_{z} \xi_{y}$ & $\frac{n}{n^{\prime}}=\frac{\sqrt{\mu_{r z} \varepsilon_{r z}}}{\xi_{z}}$ \\
& $\sqrt{\mu_{r z} \varepsilon_{r z}} \neq\left|\xi_{z}\right|$ &
\end{tabular}

and changes the sign $\left(\beta_{\mathrm{LCP}}<0\right)$ for $\xi \geq 3 * \xi_{p}$. This can be explained by the curve and sign of $\kappa_{+}$and $\kappa_{-}$shown in Figures $3(\mathrm{c})$ and $3(\mathrm{~d})$.

It is worth noting that for high values of $\xi$ and for this condition $\sqrt{\mu_{r} \varepsilon_{r}}<|\xi|$, the chiral medium behaves as a metamaterial for which the first mode becomes evanescent and the second becomes a backward wave.

3.2. Bianisotropic Case. The chirality parameter $\xi$, permeability $\mu$, and permittivity $\varepsilon$ in this case are tensors. Original results concerning the expressions of cut-off frequencies have been achieved. The particularity of these results is the possibility of controlling the specific cut-off frequencies by the choice of the adequate physical parameters. For each case, the specific cut-off frequency as function of the constitutive parameters is clearly shown in Table 2. 


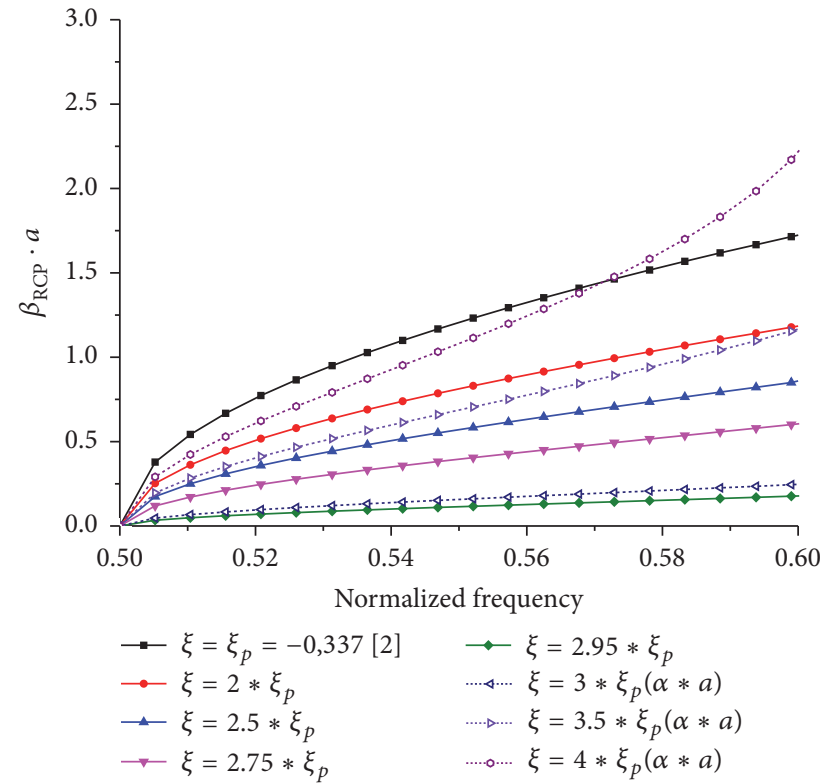

(a)

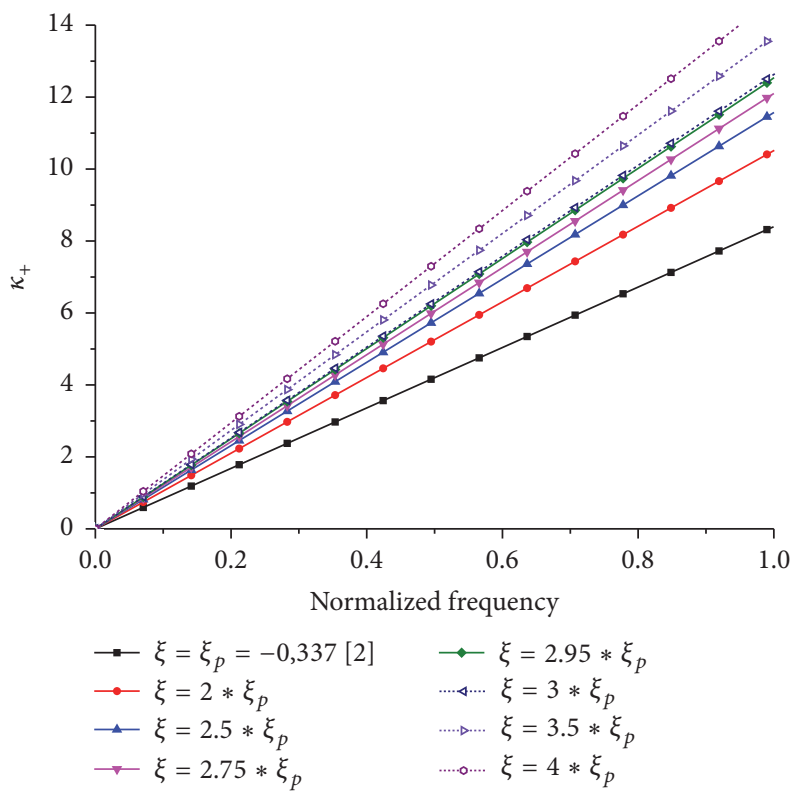

(c)

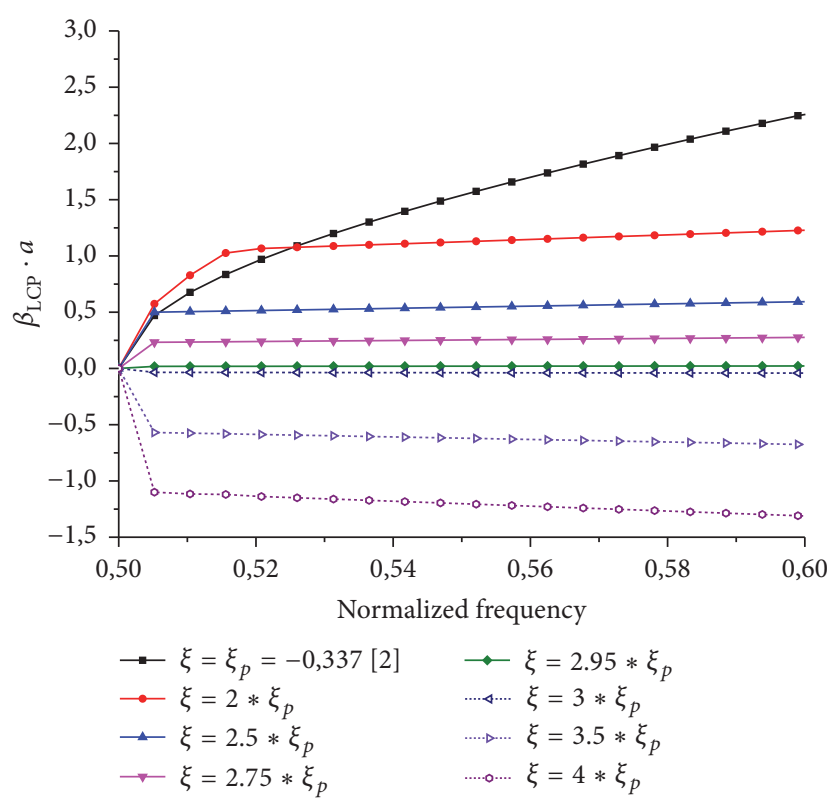

(b)

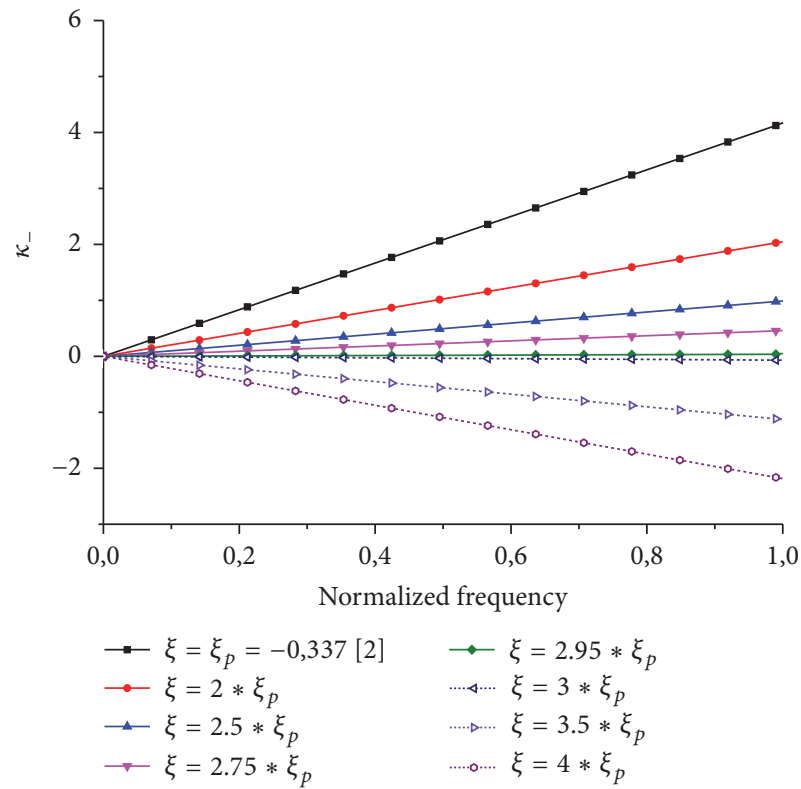

(d)

FIGURE 3: Propagation constants and wave numbers $\left(\kappa_{+}, \kappa_{-}\right)$in a parallel plate chirowaveguide in fast-fast-wave region with $\varepsilon=\varepsilon_{0}, \mu=\mu_{0}$, and $\xi$ varies. (a) RCP plane waves, (b) LCP plane waves, (c) positive wave number $\left(\kappa_{+}\right)$, and (d) negative wave number $\left(\kappa_{-}\right)$.

Our cut-off frequency calculation of the first case results in an expression function of the optic axes components $\varepsilon_{z}$ and $\mu_{z}$, which coincides with the conventional bi-isotropic formula [2], and it is obtained from bianisotropic one, when $\xi_{z} \sqrt{\mu_{r y} \varepsilon_{r y}}=\xi_{y} \sqrt{\mu_{r z} \varepsilon_{r z}}$ and $\sqrt{\mu_{r z} \varepsilon_{r z}} \neq\left|\xi_{z}\right|$. The second case results in a new and interesting expression of cutoff frequency function only of the chirality parameter $\xi_{z}$, this latter cancels the direct effect of the two parameters (permeability $\mu_{z}$ and permittivity $\varepsilon_{z}$ ) on the cut-off frequency value. The chiral parameter remains the only influencing factor. Therefore, it is easier to have much higher cut-off frequencies with low chiral parameter, leading to important and interesting results that can be used in designing optical devices such as high-pass filters. The third case is a combination of the other two cases.

Considering the conventional cut-off frequency formula obtained in the bianisotropic case (row 1 of Table 2), the effect of chirality on the propagation constant in the fast-fast-wave region is being treated through the three following examples.

(a) First Example. Taking $\left[\boldsymbol{\varepsilon}_{r}\right]=\operatorname{diag}[1,2,2],\left[\mu_{r}\right]=$ $\operatorname{diag}[1,1,1]$ and $[\xi]=\operatorname{diag}[-0.11,-0.11,-0.11]$, the condition $\sqrt{\mu_{r z} \varepsilon_{r z}}>\left|\xi_{z}\right|$ is always satisfied. 


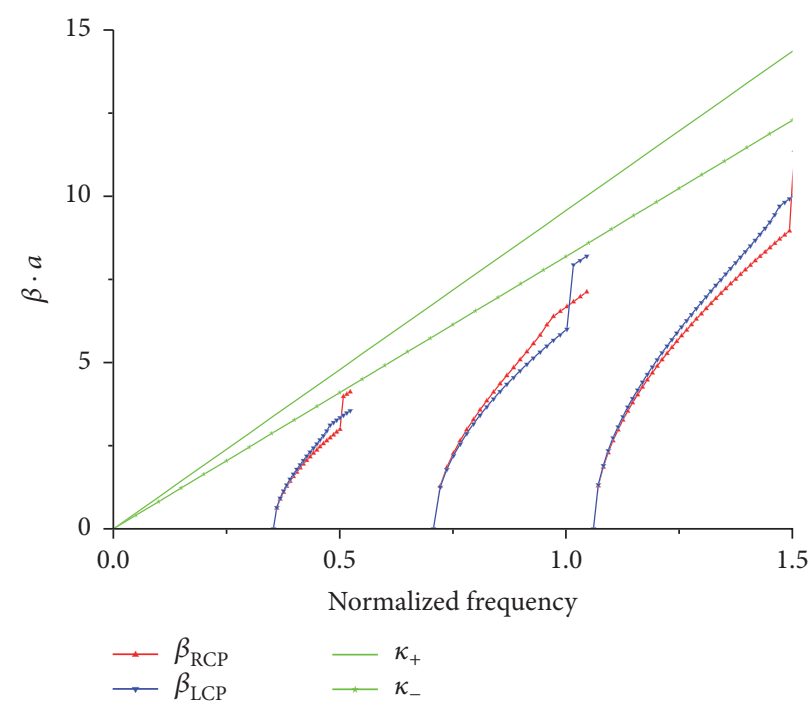

FIGURE 4: RCP and LCP propagation constants in the chirowaveguide. $\varepsilon_{r x}=1, \varepsilon_{r y}=\varepsilon_{r z}=2, \mu_{r x}=\mu_{r y}=\mu_{r z}=1$, and $\xi_{x}=\xi_{y}=\xi_{z}=-0.11$.

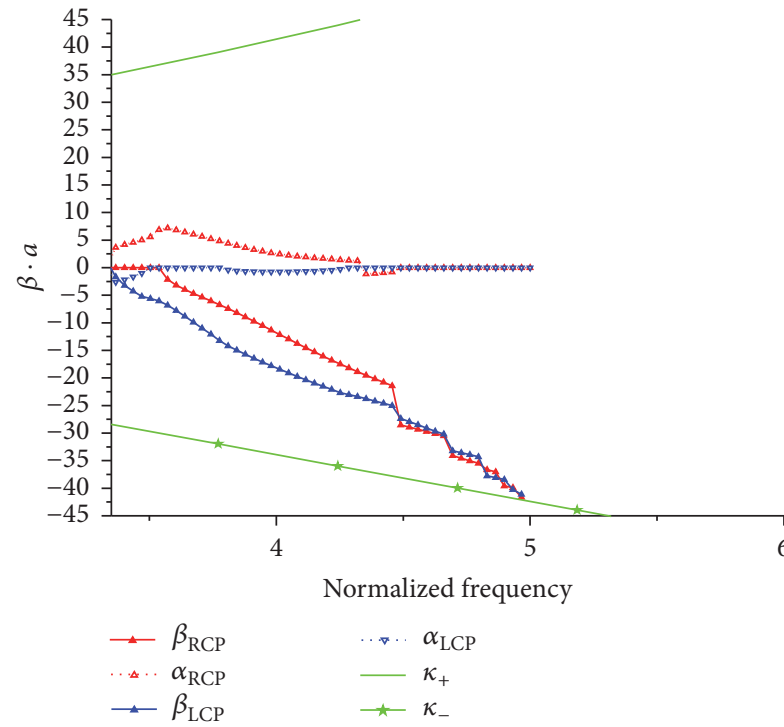

(a)

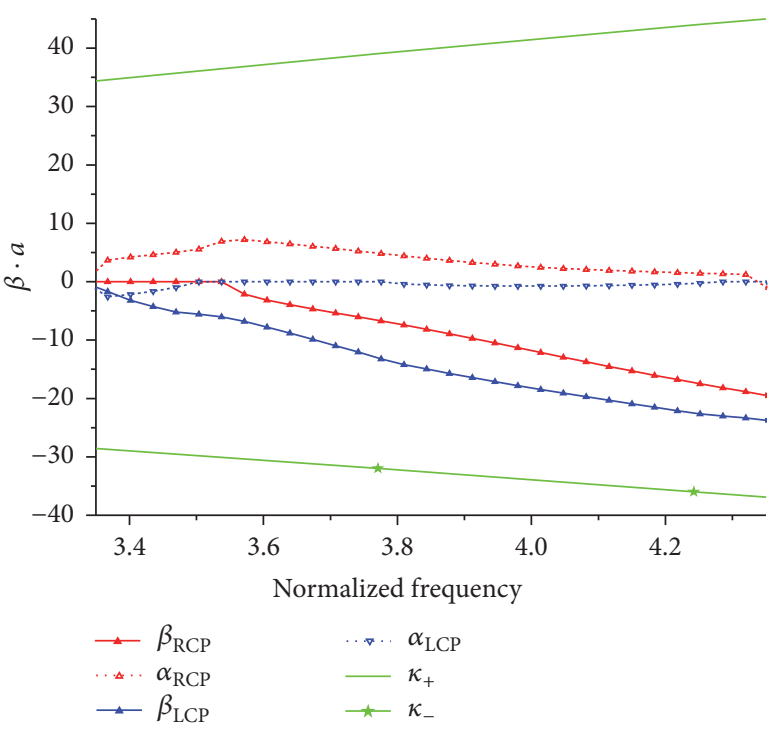

(b)

Figure 5: (a) RCP and LCP propagation constants in the chirowaveguide. (b) Zoom part of (a). $\varepsilon_{r x}=1, \varepsilon_{r y}=0.015, \varepsilon_{r z}=0.15, \mu_{r x}=1$, $\mu_{r y}=0.015, \mu_{r z}=0.15, \xi_{x}=\xi_{y}=-0.15$, and $\xi_{z}=-1.5$.

(b) Second Example. Taking $\left[\varepsilon_{r}\right]=\operatorname{diag}[1,0.15 / 10,0.15]$, $\left[\mu_{r}\right]=\operatorname{diag}[1,0.15 / 10,0.15]$, and $[\xi]=\operatorname{diag}[-0.15,-0.15$, $-10 * 0.15]$, we have $\sqrt{\mu_{r z} \varepsilon_{r z}}<\left|\xi_{z}\right|$ so that $\sqrt{\mu_{r y} \varepsilon_{r y}}<\left|\xi_{y}\right|$, because $\xi_{z} \sqrt{\mu_{r y} \varepsilon_{r y}}=\xi_{y} \sqrt{\mu_{r z} \varepsilon_{r z}}$.

(c) Third Example. Taking $\left[\varepsilon_{r}\right]=\operatorname{diag}[0.15,1,1],\left[\mu_{r}\right]=$ $\operatorname{diag}[0.15,1,1]$, and $[\xi]=\operatorname{diag}[-1.5,-0.15,-0.15]$, with $\sqrt{\mu_{r x} \varepsilon_{r x}}<\left|\xi_{x}\right|$, the condition $\sqrt{\mu_{r z} \varepsilon_{r z}}>\left|\xi_{z}\right|$ is always satisfied.

Figure 4 shows a curve of conventional RCP and LCP propagation constants even with different values of physical parameters tensors. In Figure 5, we notice that both modes behave differently even for this case. The LCP appears earlier as a backward mode $\left(\beta_{\mathrm{LCP}}<0\right)$ with RCP as an evanescent mode $\left(\alpha_{\mathrm{RCP}} \neq 0, \beta_{\mathrm{RCP}}=0\right.$, and $\alpha_{\mathrm{RCP}}$ represent the losses); then, the latter turns itself into a backward mode $\left(\alpha_{\mathrm{RCP}} \rightarrow 0\right.$ and $\left.\beta_{\mathrm{RCP}}<0\right)$. So the phase velocities of both backward modes (RCP and LCP) are negative (i.e., $\omega / \beta_{\mathrm{LCP}}<0$ and $\left.\omega / \beta_{\mathrm{RCP}}<0\right)$. This means that backward wave propagation or a negative refraction index (metamaterial medium) can be achieved using bianisotropic chiral medium with $\sqrt{\mu_{r z} \varepsilon_{r z}}<$ $\left|\xi_{z}\right|$. We notice that this result goes with the result of isotropic case presented in [18]. Figure 6 confirms that the condition $\sqrt{\mu_{r x} \varepsilon_{r x}}<\left|\xi_{x}\right|$ has no impact on the propagation nature 


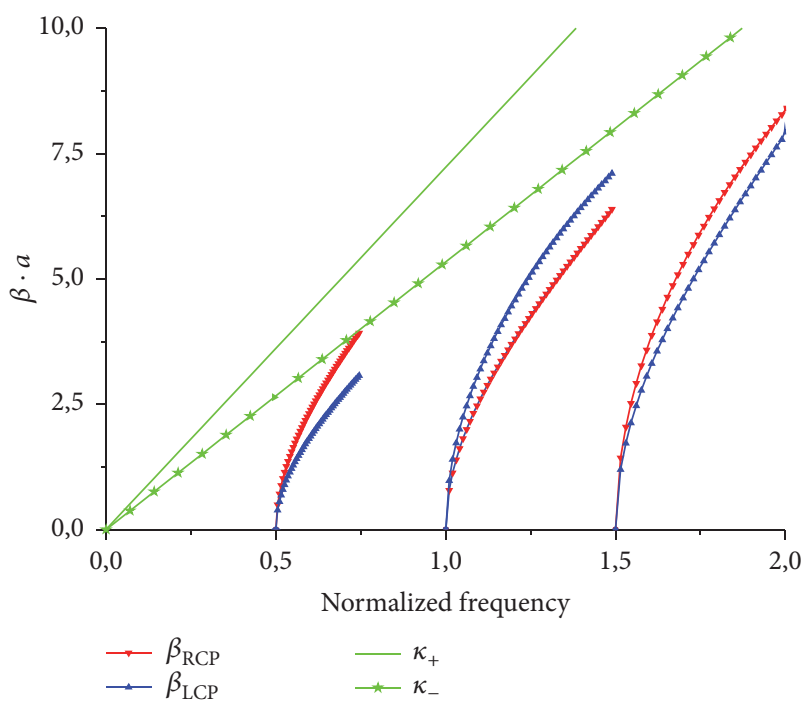

FIGURE 6: RCP and LCP propagation constants in the chirowaveguide. $\varepsilon_{r x}=0.15, \varepsilon_{r y}=\varepsilon_{r z}=1, \mu_{r x}=0.15, \mu_{r y}=\mu_{r z}=1, \xi_{x}=-1.5$, and $\xi_{y}=\xi_{z}=-0.15$.

(appearance of the backward modes). Consequently, $\xi_{z}$ is the only influencing parameter on the nature of propagating modes that allows switching from anisotropic chiral medium to metamaterial.

\section{Conclusion}

This study deals with different cases of wave propagation in parallel plate waveguide filled with anisotropic chiral medium, where three cases of study are considered using specific physical parameters. Original results of these cases have been obtained from the examination of cut-off frequencies. The first originality of this research work is the consideration of the three constitutive biaxial tensors parameters. This case of anisotropy has led to original and interesting results, where it is possible to control the specific cut-off frequencies by the choice of the adequate physical parameters. The second originality is the new calculated expression of the cut-off frequency versus the chirality in some special case. This result will undoubtedly contribute to the design of optical devices such as high-pass filters, since the effect of the chirality cancels the direct effect of the electric permittivity and magnetic permeability on the cut-off frequency expression. The third originality is the possibility of switching from the conventional anisotropic chiral medium to left-handed medium by a simple choice of the physical parameters satisfying the conditions $\sqrt{\mu_{r z} \varepsilon_{r z}}<\left|\xi_{z}\right|$, and $\xi_{z}$ is the only influencing parameter on the nature of propagating modes, the coupling parameter, and allows the switch to metamaterial.

\section{Conflicts of Interest}

The authors declare that there are no conflicts of interest regarding the publication of this paper.

\section{References}

[1] I. V. Lindell, A. H. Sihvola, S. A. Tretyakov, and A. J. Viitanen, Electromagnetic Waves in Chiral and Bi-Isotropic Media, Artech House, Boston, Mass, USA, 1994.

[2] P. Pelet and N. Engheta, “The theory of chirowaveguides," IEEE Transactions on Antennas and Propagation, vol. 38, no. 1, pp. 9098, 1990 .

[3] C. Zebiri, S. Daoudi, F. Benabdelaziz et al., "Gyro-chirality effect of bianisotropic substrate on the operational of rectangular microstrip patch antenna," International Journal of Applied Electromagnetics and Mechanics, vol. 51, no. 3, pp. 249-260, 2016.

[4] D. Sayad, F. Benabdelaziz, C. Zebiri, S. Daoudi, and R. A. AbdAlhameed, "Spectral domain analysis of gyrotropic anisotropy chiral effect on the input impedance of a printed dipole antenna," Progress in Electromagnetics Research M, vol. 51, pp. $1-8,2016$

[5] F. Ahmad, S. N. Ali, A. A. Syed, and Q. A. Naqvi, "Chiral and/or chiral nihility interfaces: parametric dependence, power tunneling and rejection," Progress In Electromagnetics Research M, vol. 23, pp. 167-180, 2012.

[6] J. B. Pendry, "Negative refraction makes a perfect lens," Physical Review Letters, vol. 85, no. 18, pp. 3966-3969, 2000.

[7] R. Fleury and A. Alù, "Cloaking and invisibility: a review (invited review)," Progress in Electromagnetics Research, vol. 147, pp. 171-202, 2014.

[8] C. Sabah, F. Dincer, M. Karaaslan, M. Bakir, E. Unal, and O. Akgol, "Biosensor applications of chiral metamaterials for marrowbone temperature sensing," Journal of Electromagnetic Waves and Applications, vol. 29, no. 17, pp. 2393-2403, 2015.

[9] N. Engheta, A. Salandrino, and A. Alù, "Circuit elements at optical frequencies: nanoinductors, nanocapacitors, and nanoresistors," Physical Review Letters, vol. 95, no. 9, Article ID 095504, 2005.

[10] J. Zhou, J. Dong, B. Wang, T. Koschny, M. Kafesaki, and C. M. Soukoulis, "Negative refractive index due to chirality," Physical Review B-Condensed Matter and Materials Physics, vol. 79, no. 12, Article ID 121104, 2009.

[11] S. Tretyakov, A. Sihvola, and L. Jylhä, "Backward-wave regime and negative refraction in chiral composites," Photonics and Nanostructures-Fundamentals and Applications, vol. 3, no. 23, pp. 107-115, 2005.

[12] B. Wang, J. Zhou, T. Koschny, M. Kafesaki, and C. M. Soukoulis, "Chiral metamaterials: simulations and experiments", Journal of Optics A: Pure and Applied Optics, vol. 11, no. 11, Article ID 114003, 2009.

[13] S. Tretyakov, I. Nefedov, A. Sihvola, S. Maslovski, and C. Simovski, "Waves and energy in chiral nihility," Journal of Electromagnetic Waves and Applications, vol. 17, no. 5, pp. 695-706, 2003.

[14] J. B. Pendry, "A chiral route to negative refraction," Science, vol. 306, no. 5700, pp. 1353-1355, 2004.

[15] S. Ougier, I. Chenerie, A. Sihvola, and A. Priou, "Propagation in bi-isotropic media: effect of different formalisms on the propagation analysis," Progress in Electromagnetics Research, vol. 9, pp. 19-30, 1994.

[16] A. Ghaffar and M. A. S. Alkanhal, "Electromagnetic waves in parallel plate uniaxial anisotropic chiral waveguides," Optical Materials Express, vol. 4, no. 9, pp. 1756-1761, 2014. 
[17] A. L. Topa, C. R. Paiva, and A. M. Barbosa, "Electromagnetic wave propagation in chiral H-guides," Progress in Electromagnetics Research, vol. 103, pp. 285-303, 2010.

[18] C. Sabah, "Left-handed chiral metamaterials," Central European Journal of Physics, vol. 6, no. 4, pp. 872-878, 2008. 


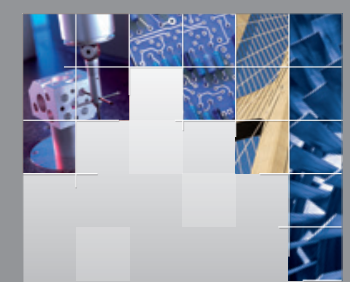

\section{Enfincering}
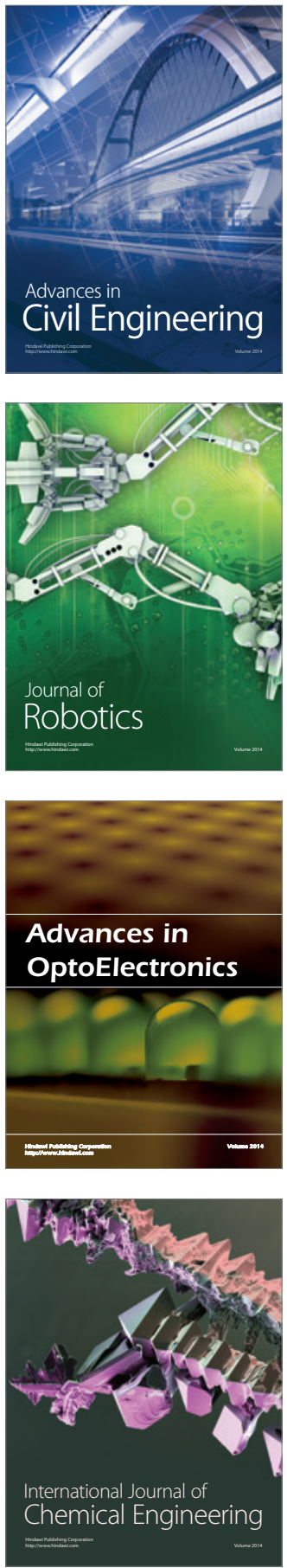

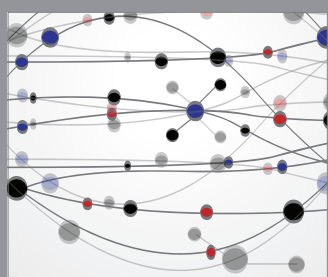

The Scientific World Journal

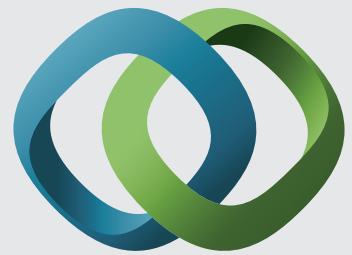

\section{Hindawi}

Submit your manuscripts at

https://www.hindawi.com
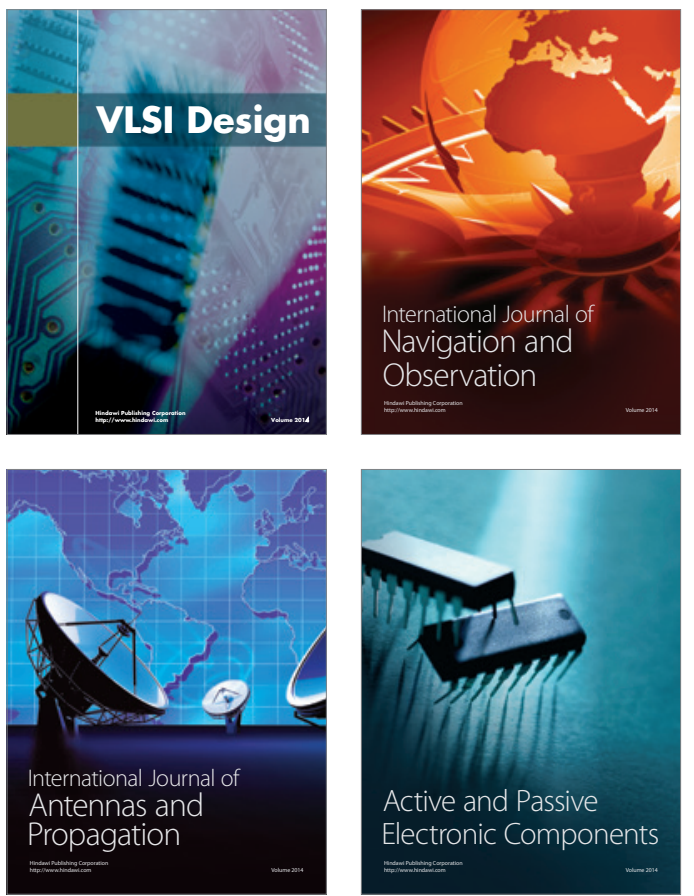
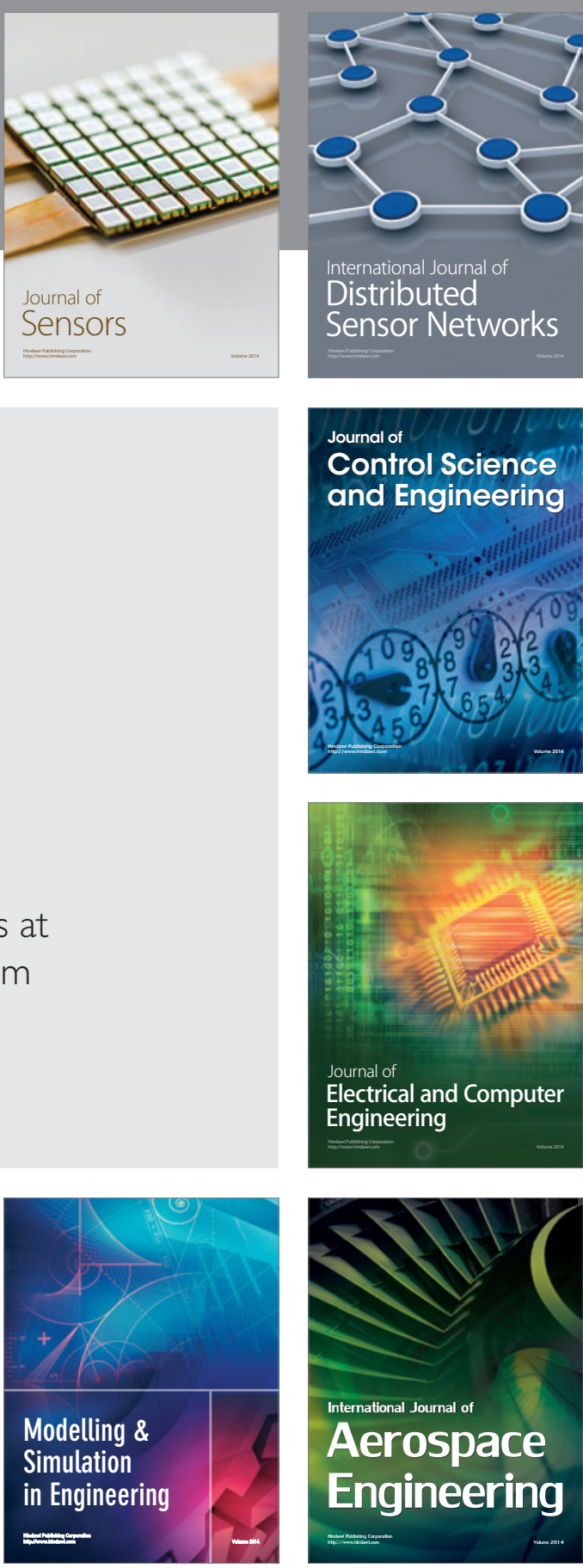

International Journal of

Distributed

Sensor Networks

$-$

Joumal of

Control Science

and Engineering
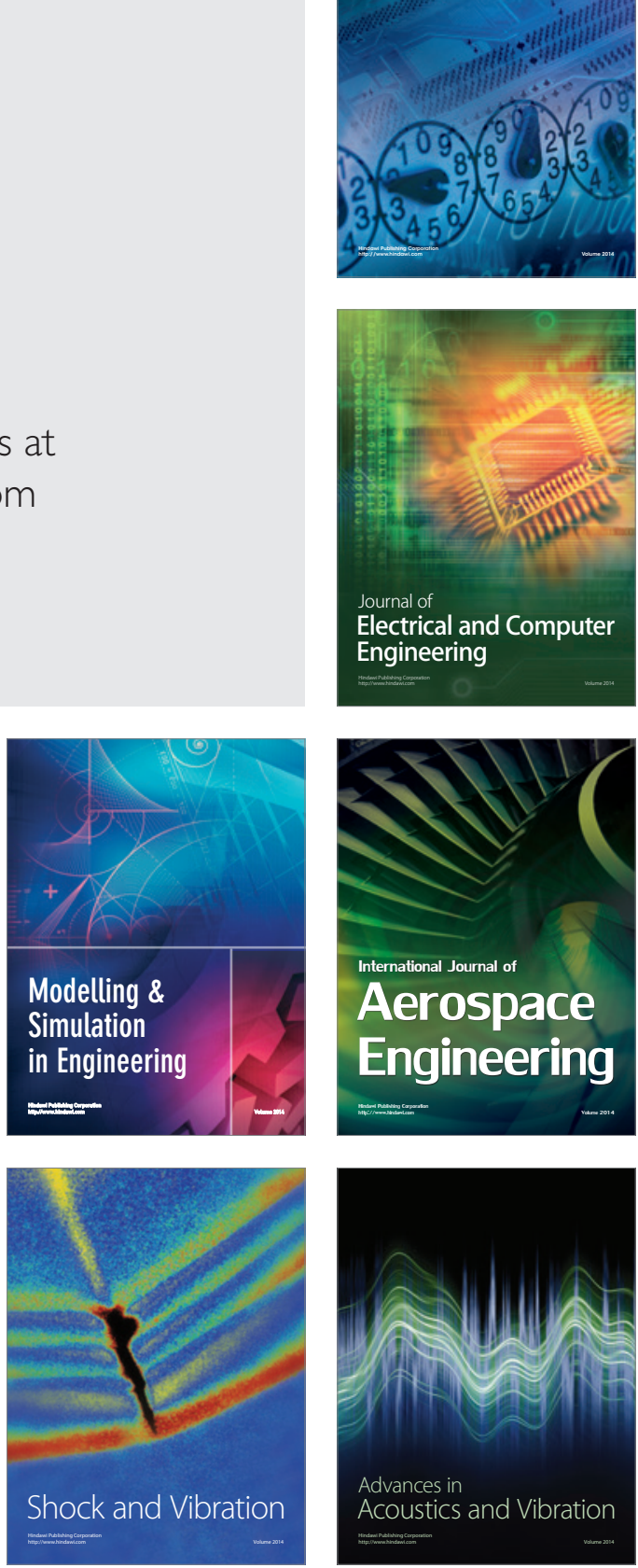